\title{
Measurement of the longitudinal spin structure of the proton at COMPASS
}

\author{
Elena Zemlyanichkina*(on behalf of COMPASS collaboration) \\ Joint Institute for Nuclear Research \\ E-mail: Elena.Zemlyanichkina@cern.ch
}

Inclusive $\left(A_{1, p}\right)$ and semi-inclusive $\left(A_{1, p}^{\pi+}, A_{1, p}^{\pi-}, A_{1, p}^{K+}\right.$ and $\left.A_{1, p}^{K-}\right)$ double-spin asymmetries in deepinelastic muon-proton scattering have been measured at COMPASS (SPS CERN). The results have been obtained with data collected in 2007 for the longitudinal polarised $\mathrm{NH}_{3}$ solid target and beam of positive muons with energy $E=160 \mathrm{GeV}$.

We improve the statistical precision of $g_{1}^{p}\left(x \cdot Q^{2}\right)$ by a factor of two in the low $x$ region. Proton asymmetries have been combined with published deuteron ones to extract the non-singlet spin-dependent structure function $g_{1}^{N S}\left(x, Q^{2}\right)$. The first moment $\Gamma_{1}^{N S}$ confirms the validity of the Bjorken sum-rule.

Our new semi-inclusive data were used to evaluate the $\Delta u, \Delta d, \Delta \bar{u}, \Delta \bar{d}$ and $\Delta s \equiv \Delta \bar{s}$ distributions.

XVIII International Workshop on Deep-Inelastic Scattering and Related Subjects, DIS 2010

April 19-23, 2010

Firenze, Italy

\footnotetext{
* Speaker.
} 


\section{Introduction}

The proton data presented in this paper were collected with the COMPASS [1] spectrometer during the year 2007.

All events are required to have a reconstructed primary interaction vertex defined by the incoming and the scattered muon trajectories. For the canceling of the muon flux in the asymmetry calculation the trajectory of an incoming muon with an energy in the interval $140<E_{\mu}<180 \mathrm{GeV}$ is required to cross all target cells. The kinematic region is defined by cuts on the photon virtuality, $\mathrm{Q}^{2}>1(\mathrm{GeV} / \mathrm{c})^{2}$, and the fractional energy, $0.1<y<0.9$. The results on the spin-dependent structure function $g_{1}^{p}\left(x, Q^{2}\right)$ [2] have been combined with the $g_{1}^{d}\left(x, Q^{2}\right)$ ones [3] to evaluate the non-singlet structure function $g_{1}^{N S}$.

In this paper also we present an evaluation of the helicity quark distribution $\Delta u, \Delta d, \Delta \bar{u}, \Delta \bar{d}$ and $\Delta s(=\Delta \bar{s})$. They were obtained in a combined analysis of inclusive and identified hadron asymmetries. Both deuteron [4] and proton data have been used.

The hadron from the main vertex is required to carry a fractional energy $0.20<z<0.85$ and must be identified as a pion or a kaon by the RICH detector, which limits its momentum to the range $10<p<50 \mathrm{GeV} / \mathrm{c}$. The total statistics of samples obtained with the proton target for the inclusive, $\pi^{+}\left(\pi^{-}\right)$and $\mathrm{K}^{+}\left(\mathrm{K}^{-}\right)$events after all cuts is $85.3,13.3(11.8)$ and 3.9(2.6) million events, respectively.

Since the samples of identified pions and kaons do not fully correspond to the true ones, an unfolding procedure had been applied to correct rates and asymmetries. The purity of the pion samples selected by the RICH detector is above 0.98 over the full range of $x$. For the kaons it varies from about 0.73 at the lowest value of $x$ to about 0.93 and 0.91 at $x \geq 0.03$ for positive and negative kaons, respectively. The corresponding unfolding correction has only a small effect on the pion and kaon asymmetries.

\section{Structure function $g_{1}^{p}$ and NLO QCD fit of $g_{1}^{N S}$}

The longitudinal virtual-photon-proton asymmetry $A_{1}^{p}$ (Fig. 1) is evaluated from the numbers of events collected in the different target cells by the method used in our previous analysis of deuteron data [3, 4]. The longitudinal spin structure function $g_{1}^{p}$ of the proton is obtained from $A_{1}^{p}$ using the relation:

$$
g_{1}^{p}=\frac{F_{2}^{p}}{2 x(1+R)} A_{1}^{p}
$$

where $F_{2}^{p}$ and $R$ are the spin-independent structure function. $g_{1}^{p}$ as a function of $x$ is shown in Fig. 2 (left).

Good and comparable precision at low $x$ for results on $g_{1}^{d}$ and $g_{1}^{p}$ gives ideal condition for a new evaluation of the non-singlet structure function

$$
g_{1}^{N S}(x)=g_{1}^{p}(x)-g_{1}^{n}(x)=2\left[g_{1}^{p}(x)-\frac{g_{1}^{d}(x)}{\left(1-3 / 2 \omega_{D}\right)}\right]
$$

where $\omega_{D}$ is the deuteron D-state probability $\left(\omega_{D}=0.05 \pm 0.01\right)$. 


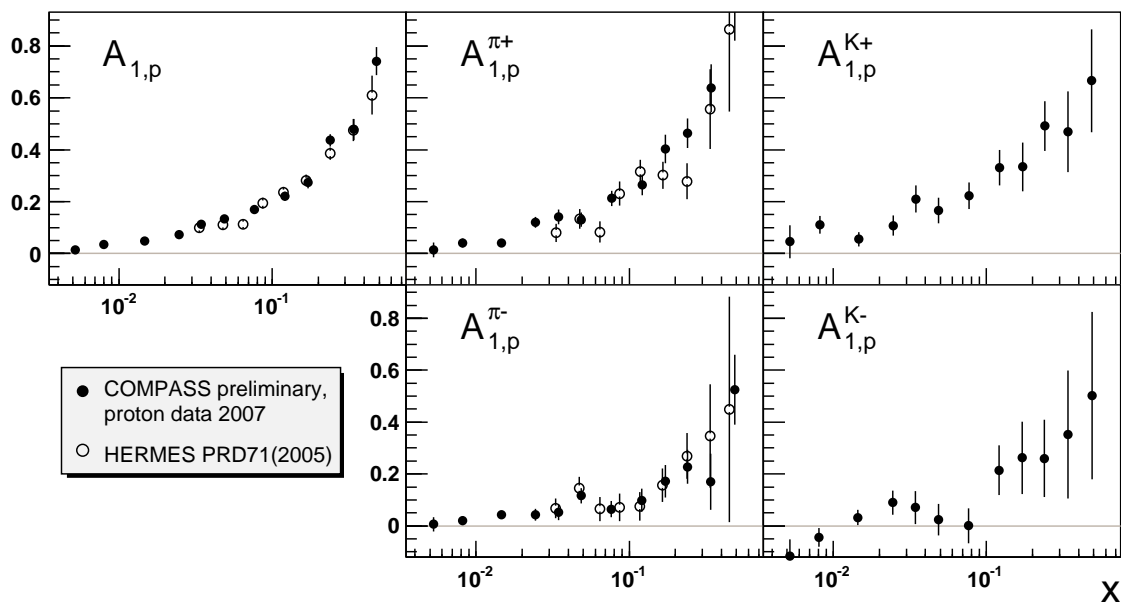

Figure 1: Comparison of double-spin asymmetries of COMPASS as a function of $x$ with results of HERMES [5].

The first moment provides a test of the Bjorken sum rule, a fundamental result of QCD derived using current algebra

$$
\int_{0}^{1} g_{1}^{N S}(x) d x=\frac{1}{6}\left|\frac{g_{A}}{g_{V}}\right| C^{N S}
$$

The $Q^{2}$ dependence of the non-singlet structure function $g_{1}^{N S}(x)$ is decoupled from the singlet quark and the gluon spin densities. Consequently a fit of the $Q^{2}$ evolution of source requires only a small number of parameters to describe the shape of $\Delta q_{3}(x)$ at some reference $Q^{2}$. In the present analysis, $Q_{0}^{2}=3(\mathrm{GeV} / c)^{2}$ is taken as reference $Q^{2}$ in the fit and the following parameterisation used for $\Delta q_{3}$ :

$$
\Delta q_{3}(x)=\eta_{3} \frac{x^{\alpha_{3}}(1-x)^{\beta_{3}}}{\int_{0}^{1} x^{\alpha_{3}}(1-x)^{\beta_{3}} d x} .
$$

Results of the fit are shown in Fig. 2 (right). Value of $\left|g_{A} / g_{V}\right|$ was obtained using Eq. 2.3:

$$
\left.\left|g_{A} / g_{V}\right|=1.28 \pm 0.07 \text { (stat. }\right) \pm 0.10 \text { (syst.) }
$$

The dominant systematic error is due to uncertainties on the beam and target polarisation.

\section{Polarized PDFs from fit to the asymmetries}

At LO in QCD under the assumption of independent quark fragmentation, the double spin asymmetries for a hadron $h$ produced in the current fragmentation region can be decomposed into a sum of products of quark helicity distributions $\Delta q\left(x, \mathrm{Q}^{2}\right)$ times quark fragmentation functions $D_{q}^{h}\left(z, \mathrm{Q}^{2}\right)$, where $z$ is the fraction of the virtual photon energy taken by the hadron $h$ :

$$
\mathrm{A}_{1}^{h}\left(x, \mathrm{Q}^{2}, z\right)=\frac{\sum_{q} e_{q}^{2} \Delta q\left(x, \mathrm{Q}^{2}\right) D_{q}^{h}\left(z, \mathrm{Q}^{2}\right)}{\sum_{q} e_{q}^{2} q\left(x, \mathrm{Q}^{2}\right) D_{q}^{h}\left(z, \mathrm{Q}^{2}\right)}
$$



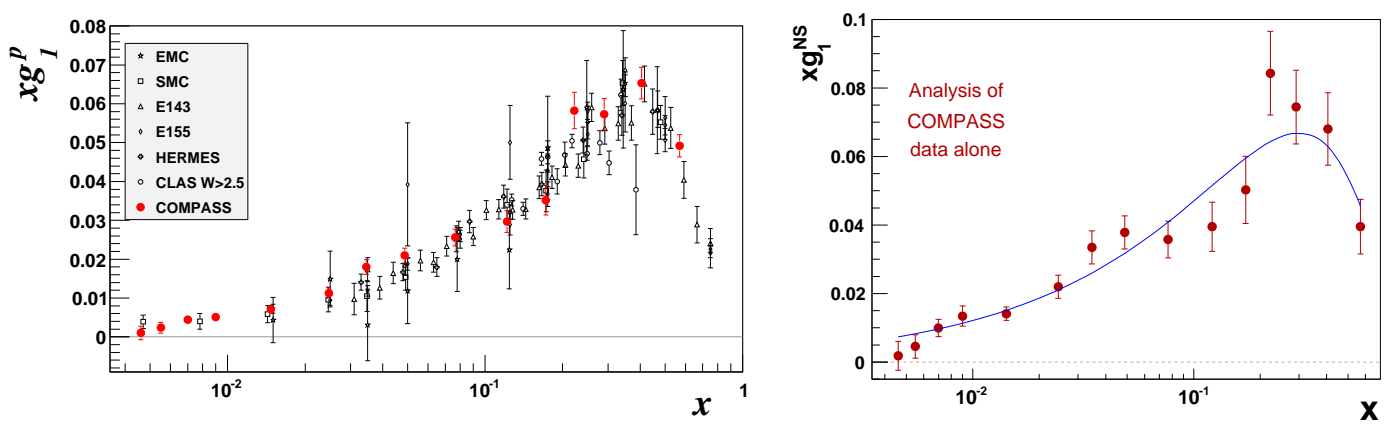

Figure 2: (left) $g_{1}^{p}$ as a function of $x$ [2]; (right) Values of $g_{1}^{N S}(x)$ at $Q_{0}^{2}=3(\mathrm{GeV} / c)^{2}$. The curve was obtained in the QCD NLO fit of the COMPASS data.

In the present analysis we neglect the $Q^{2}$ dependence of the asymmetries and assume all measurements to be valid at $Q_{0}^{2}=3(\mathrm{GeV} / c)^{2}$. For the unpolarised parton distributions we use the LO parametrisation of MRST [6] and for fragmentation functions the LO parametrisation of DSS [7].

The four semi-inclusive asymmetries on the proton, the four on the deuteron and the two inclusive asymmetries provide a system of ten equations with five unknowns $(\Delta u, \Delta d, \Delta \bar{u}, \Delta \bar{d}$ and $\Delta s \equiv \Delta \bar{s})$. The equations are solved by a least-square fit, independently in each bin of $x$. The analysis is limited to $x \leq 0.3$ because sea quarks contributions become insignificant above this limit. The results of the fit are shown on the Fig. 3.

Presently $\Delta \bar{u}-\Delta \bar{d}$ is accessible only via semi-inclusive DIS processes. The flavour asymmetry in the helicity distribution of the sea $\Delta \bar{u}-\Delta \bar{d}$ is shown in Fig. 4 in comparison with HERMES results of Ref. [5]. The more precise COMPASS results indicate a slightly positive distribution. The first moment of the distribution in the $x$ region of the measurement is

$$
\int_{0.004}^{0.3}(\Delta \bar{u}-\Delta \bar{d}) d x=0.052 \pm 0.035 \text { (stat.) } \pm 0.013 \text { (syst.) . }
$$

New COMPASS data on longitudinal polarized $\mathrm{NH}_{3}$ target will be collected during the year 2011, they will allow to increase the precision of the measurements.

\section{References}

[1] P. Abbon et al. [COMPASS Collaboration], Nucl. Instrum. Meth. A 577 (2007) 455 [arXiv:hep-ex/0703049].

[2] M. G. Alekseev et al. [The COMPASS Collaboration], arXiv:1001.4654 [hep-ex].

[3] V. Y. Alexakhin et al. [COMPASS Collaboration], Phys. Lett. B 647 (2007) 8 [arXiv:hep-ex/0609038].

[4] M. Alekseev et al. [COMPASS Collaboration], Phys. Lett. B 680 (2009) 217 [arXiv:0905.2828 [hep-ex]].

[5] A. Airapetian et al. [HERMES Collaboration], Phys. Rev. D 71 (2005) 012003 [arXiv:hep-ex/0407032].

[6] A. D. Martin, W. J. Stirling and R. S. Thorne, Phys. Lett. B 636 (2006) 259 [arXiv:hep-ph/0603143].

[7] D. de Florian, R. Sassot and M. Stratmann, Phys. Rev. D 75 (2007) 114010 [arXiv:hep-ph/0703242]. 


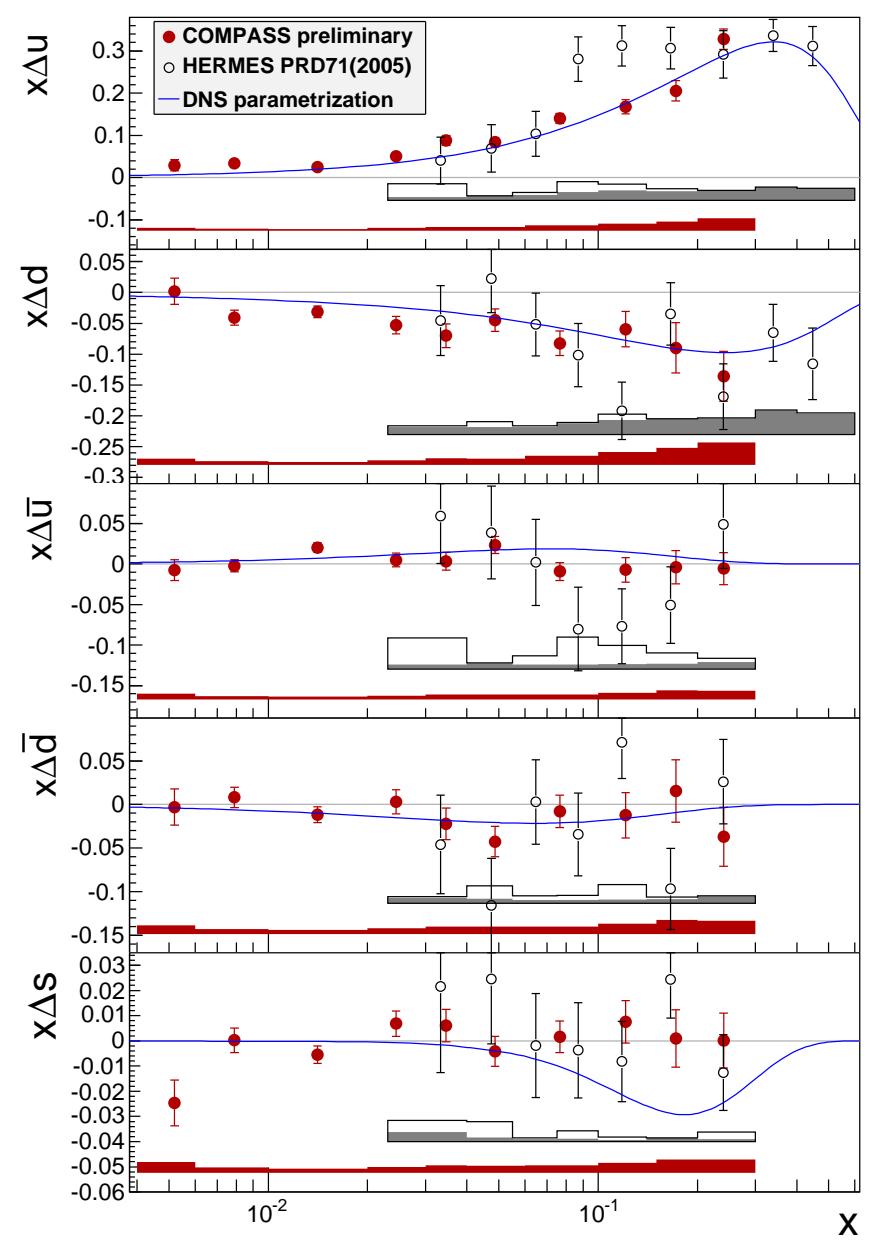

Figure 3: The quark helicity distributions as a function of $x$.

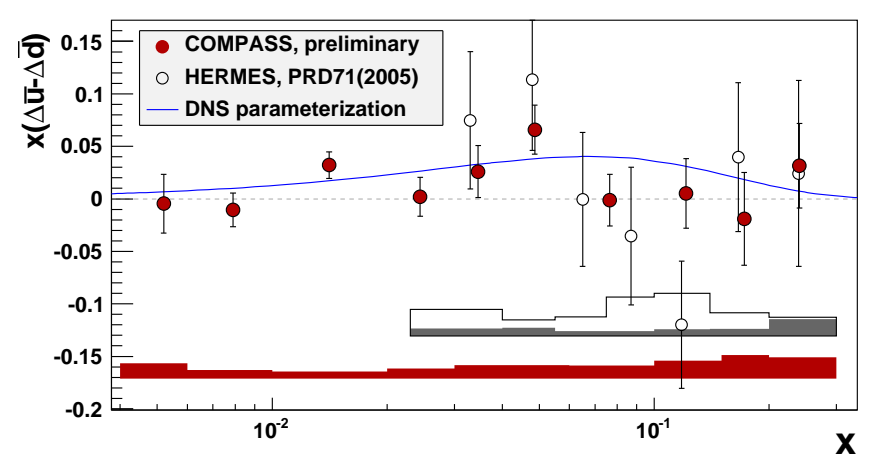

Figure 4: The flavour asymmetry of the helicity distribution of the sea $x \cdot(\Delta \bar{u}-\Delta \bar{d})$ mesuared by COMPASS at $Q_{0}^{2}=3(\mathrm{GeV} / c)^{2}$ and by HERMES at $Q_{0}^{2}=2.5(\mathrm{GeV} / c)^{2}$. 